\section{RESPONSIBLE LABS FOR DENTISTS}

DAMAS is currently the only independently audited 'Quality Management System' that can prove a dental laboratory complies with the Medical Devices Directive (MDD). DAMAS registered dental laboratories have taken steps to ensure you have "peace of mind' for yourself and your patients.

Laboratories wishing to become registered with DAMAS have to undergo a stringent auditing process and implement regular checks throughout every stage of their production process. DAMAS is based on proven quality management principles and is tailored to reflect the practical aspects of manufacturing dental appliances and compliance with dental industry regulations.

By choosing a DAMAS laboratory you will be working alongside a laboratory that is proactive, interested in quality measures and serious about their business. These laboratories have invested in their business to provide their clients with exceptional levels of service. The laboratory you use will be a reflection of the treatments you provide to your patients.

Reader response number 59

\section{SIMPLE TREATMENT, GREAT RESULTS}

One of the leading trends in cosmetic dentistry in recent years has been adult orthodontics. Patients keen to emulate their favourite stars are opting for minimally invasive treatments that will give them the smile they desire.

What every patient wants is an orthodontic treatment that is simple, effective, fast and discreet and with the Oralign system, that's exactly what they get.

Launched in May 2011, the Oralign orthodontic appliance uses a unique method of interdental stripping and a small, discreet removable appliance to resolve the problem of overcrowding in

\section{CONTROLLED MEMORY FILE}

Coltène Whaledent has launched Hyflex $\mathrm{CM}$, the controlled memory NiTi file which, because of no memory effect, is extremely flexible to adapt to canal anatomy to significantly reduce the risk

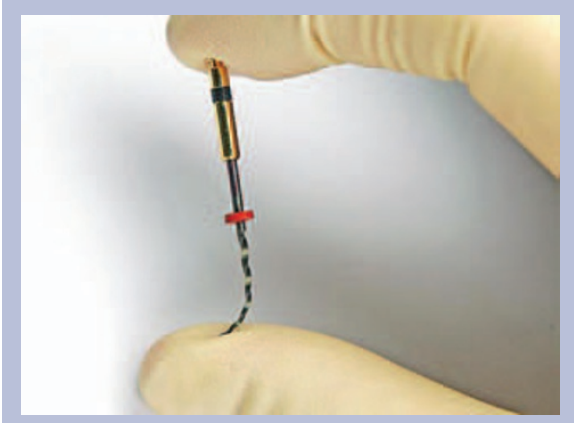

one or both arches, without affecting the rest of the mouth.

Oralign technicians use X-rays, photos and full mouth impressions taken during consultation to provide you with costing, a treatment plan and a projected outcome. You can then offer your patients the appliance, which can straighten teeth in less than 14 weeks when worn for just 14 hours a day including overnight. The retainer that accompanies the appliance is then fixed in position to prevent any relapse.

Providing excellent results in a short space of time, patients really have something to smile about with the new Oralign orthodontic appliance.

Reader response number 60

of perforation of the canal. This also allows pre-bending similar to stainless steel files and is perfect for extremely curved root canals.

Hyflex CM NiTi files are multi-use and with autoclaving or with a glass bead steriliser the files regain the original shape whilst also strengthening the file and with controlled memory are up to $300 \%$ more resistant to cyclical fatigue compared to conventional NiTi Files which substantially helps reducing the incidence of file separation. This is an amazing new addition to the Coltène Whaledent endodontic portfolio.

Reader response number 61

\section{MORE THAN IMPLANTS}

The Straumann Dental Implant System has been developed to offer treatment flexibility, so you can use the same surgical kit, prosthetic kit and procedure whichever Straumann Implant is demanded by the clinical situation. The essential conditions required for implant dentistry can successfully be achieved with their range of regenerative products, providing dentists with solutions for true soft- and hardtissue regeneration that are backed by scientific evidence.

Now, Straumann's product portfolio encompasses not only dental implants and oral tissue regeneration but digital solutions. Straumann CARES Digital Solutions encompasses stateof-the-art computer- guided surgery, intra-oral scanning, and CADCAM prosthetics bringing high tech digital solutions to the heart of the dental practice and laboratory.

Reader response number 62

\section{NOTHING LEFT TO CHANCE}

The Bien-Air Chiropro L implantology system leaves nothing to chance.

Its intuitive interface can be managed by the control pedal. The patented peristaltic pump with disposable irrigation lines and insertion support ensures easy handling and optimum infection control.

The Chiropro L System includes the MX-LED self-ventilating motor, which is the most powerful on the market to offer LED lighting at equal intensity at both high and low speeds. Incorporating seven of the leading brands of implants with their complete sequences the Chiropro L can also be customised to your individual requirements.

With the world's first internal irrigation system the 20:1 contra-angle handpiece incorporates the smallest head on the market allowing access to the tightest of areas. Fitted with an exclusive double optical glass rod system, the handpiece provides uniform lighting of the operative field. This user-friendly system has proved to meet the demands of implantologists worldwide and more. Reader response number 63 